\title{
Frontières
}

\section{Levé, Édouard, Suicide, Paris, P.O.L éditeur, 2008, 123 p.}

\section{Shahira El Moutei-Khalil}

Volume 21, numéro 1, automne 2008

Prévenir le suicide

URI : https://id.erudit.org/iderudit/037890ar

DOI : https://doi.org/10.7202/037890ar

Aller au sommaire du numéro

Éditeur(s)

Université du Québec à Montréal

ISSN

1180-3479 (imprimé)

1916-0976 (numérique)

Découvrir la revue

Citer ce compte rendu

Moutei-Khalil, S. E. (2008). Compte rendu de [Levé, Édouard, Suicide, Paris, P.O.L éditeur, 2008, 123 p.] Frontières, 21(1), 144-145.

https://doi.org/10.7202/037890ar

Ce document est protégé par la loi sur le droit d'auteur. L'utilisation des services d'Érudit (y compris la reproduction) est assujettie à sa politique d'utilisation que vous pouvez consulter en ligne.

https://apropos.erudit.org/fr/usagers/politique-dutilisation/
Cet article est diffusé et préservé par Érudit.

Érudit est un consortium interuniversitaire sans but lucratif composé de l’Université de Montréal, l'Université Laval et l'Université du Québec à Montréal. Il a pour mission la promotion et la valorisation de la recherche. https://www.erudit.org/fr/ 


\section{VILLANI, Arnaud}

\section{Petites méditations métaphysiques sur la vie et la mort}

Paris, Hermann éditeurs, 127 p.

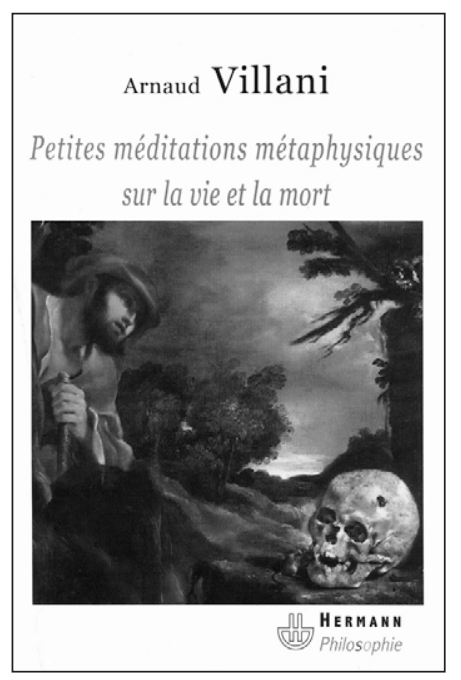

Douze chapitres courts mais denses, douze méditations sur la vie, la mort, la vie-mort, la mortvie, un discours inspiré d'autres philosophes et inspirant à son tour, unique et juste dans son expression, voilà qui présente en quelques mots l'œuvre de Villani. Déjà dans la dédicace faite à son père décédé, l'auteur nous invite à considérer la mort comme une vie: «À mon père, pour la première année de sa nouvelle vie de mort. » II se propose de nous montrer "que la mort est tout le contraire de rien ». Et voilà, avec A. Villani, nous entreprenons une série de méditations dont j'essaierai de rendre compte, selon l'entendement que j'en ai, selon la lecture volontairement sélective et conséquemment limitée que j'en fais.

La première méditation nous amène à réfléchir sur la grande solitude de l'humain, élément d'un ensemble constitué de parties à la fois semblables et différentes où chacun doit assumer sa responsabilité, donner sens à la succession de ses actes. La mort s'affronte seul, c'est un moment d'abandon pur. Et pour finir, nous mourrons tous. "II aura sans doute été inutile, insensé de se battre pour qu'une poussière prenne cette forme, pour qu'un dépôt d'étoile finisse "moi" » (p. 15). L'individu est un et à la fois multitude; quand il meurt, le monde se lève une dernière fois pour s'amuir [sic] « dans la poudre des siècles». La vie aura été une recherche à tâtons de ce qui est vivant, même si au bout du compte, cela "prend la forme d'une inquiétante étrangeté».
Quand nous mourons, nous emportons un peu ou beaucoup de ceux qui nous étaient attachés, les faisant ressembler dans leur deuil "à des épouvantails» mais, tout de même, la vie continue sans nous. Chacun a la charge de trouver le chemin qui manque à l'humanité, de se frayer un chemin, un chemin de terre et sur Terre, en signe de croix: sur l'horizontale avec ses frères (ses contemporains) (corps et terre) et sur la verticale avec ses pères (ses racines) (esprit). Le Christ, selon Villani, n'est pas le seul à donner sens à la croix. "Ce qui crucifie I'homme, [...] c'est un corps lourd qui vient de la terre et y retourne [...] c'est un esprit comme ligne d'éther [...] venant croiser la ligne de fond des corps et de la terre" (p. 24). C'est ce que je retiens de la deuxième méditation. Que signifie «je» au moment de la mort? La troisième méditation porte, du moins en partie, sur la fragilité de la vie et sur l'impermanence des liens. "Tout attachement est désirable par l'effet de son plus secret détachement» (p. 35). La mort donne à la vie son éclat. Quelle passion mettrions-nous à une vie, à un monde éternellement donnés?

La quatrième méditation en sera une sur la vieillesse, les "heurs" "bon-heurs» "mal-heurs» de la vieillesse. Ce qui donne sens à la vie, ce sont nos amours, nos liens, nos relations. Ce que nous avons fait perd de la valeur au fur et à mesure des années. Pourtant, nous conservons un inlassable appétit de vivre. «Mais n'allons pas croire qu'il n'y ait que souffrance dans le vieillissement. Rien ne donne une meilleure gustation du réel. Derrière chaque geste, on sent le souvenir de tous les précédents" (p. 40). Et chez les vieillards, la rage de douleur témoigne de la sensibilité, de la vie restantes. Le seul possible, c'est continuer à vivre même souffrants, se rattacher à des riens, croire et faire croire à la vie; le seul impossible, ne pas mourir. La mort nous apprend qu'il faut faire vivre la vie. Voilà l'objet de la cinquième méditation. De la sixième méditation, je retiens pour vous que "chaque instant [...] déplace hors temps et hors espace, loin de tout repère, l'âme et la mort» et que "ne pas espérer, c'est manquer aussi l'inespéré » (p. 58). Dans la septième méditation, Villani évoque la tension continue entre la vie et la mort; cette tension crée la vie. Il compare cette tension à une tente qu'il appartient à l'être de maintenir déployée.

Lors de la huitième méditation, l'auteur aborde le décès de son père, le langage des mains. "II me serre, me presse, m'imprime. II me transmet - quoi ? un signe, un avertissement, une connivence, un appel, une demande pressante, la sigillée de la dépendance, l'empreinte de l'être qui le quitte?» (p. 75) II présente son carrefour des trois mains: la première, celle du père mourant, la deuxième, la sienne qui est tendue à son père pour "l'aider à passer le pas", la troisième celle de son nouveau-né qui ne lâche plus l'objet tombé sous sa main. II sent la même emprise sur la vie chez le nouveauné et l'aïeul et y perçoit la même vie. Penser la mort en philosophie peut aider à tenir loin la $9^{\mathrm{e}}$ heure du "Je", moment de solitude et de macération. Il est possible qu'en mourant je laisse les proches sans protection mais cela les forcera à desserrer l'emprise, à occuper l'espace laissé vacant, à grandir.

La neuvième méditation nous invite à réfléchir sur le geste le plus divin qui soit: ouvrir grand ses bras pour accueillir l'autre comme le Christ le fit sur la croix pour accueillir I'humanité tandis que ce qui calme la brûlure de la mort, c'est de penser que tout arrive en son temps, est à sa place. Une âme est riche de la multitude de ses attaches. "Une âme commence à naître ( $d$ 'après l'auteur, on n'a pas d'âme en naissant, on se la fait) lorsque les liens avec le monde se croisent assez pour la sustenter» (p. 99). L'âme file, tisse des liens avec le monde selon ses désirs ou aspirations; par contre, la mort serait la défileuse, I'anti-fileuse, la défilante. "Elle est la manie de se défiler. " À ma mort, je récapitulerai mes luttes entre la terre et l'esprit. Et, à bien y penser, il doit y avoir une immortalité de l'âme individuelle, elle est exactement la place "que définissent les liens tissés " pour de vrai ", dès cette vie » (p. 102). La mort est vérité en ce qu'elle donne de force et de lucidité pour vivre en plus grand. Ce sont là des fragments de la dixième méditation.

La onzième méditation amène le méditant à dire: finalement, la mort ne change pas grand-chose, le type de mouvement redevient atomique, constituant le fond des choses; elle ne change rien sinon l'échelle de temps. "Les choses ne sont ni ainsi ni autrement mais précisément ainsi et autrement, ailleurs tout comme ici, métamorphose qui est le sens de l'univers» (p. 115). Dans la douzième méditation, l'auteur qui a affirmé que la mort ne change rien à rien, qui a voulu être simple et simplifier doit ici se reprendre: «la mort exprime celui qui s'exprime et n'en laisse pas une goutte. Elle le pousse à bout, le miniaturise ». " [...] [L]'étonnante collection qu'était devenu l'homme collige maintenant la très simple poussière » (p. 120121). La mort s'abstient de détails car le détail donne vie. Avec elle, nous ne sommes pas seulement jetés dans l'infini, nous lui sommes intérieurs. Ce qui caractérise le cosmos dont l'humain fait partie, c'est le mouvement directionnel sans arrêts, de l'un à l'autre, de la vie à la mort; voilà l'appel.

Le sens de ces douze méditations de Villani ne se laisse pas saisir d'emblée par le profane. J'aurais souhaité rendre davantage justice à l'auteur mais plusieurs lectures ou, plus précisément, plusieurs méditations sur chacune de ses douze méditations m'auraient été nécessaires. Quoi qu'il en soit, ces méditations très philosophiques sont d'abord et avant tout une invitation à apprivoiser la mort, à considérer notre passage sur cette terre pour ce qu'il est et à reconnaître indubitablement l'importance des liens que nous tissons et des détails que nous créons qui donnent vie à la vie et écartent, dans l'ici et maintenant, la mort.

\section{Denise Badeau}

\section{LEVÉ, Édouard}

\section{Suicide}

Paris, P.O.L éditeur, 2008, 123 p.

Édouard Levé était à la fois peintre, photographe et écrivain; il s'est donné la mort le 15 octobre 2007, à l'âge de 42 ans. Trois jours plus tôt, il avait remis à son éditeur un manuscrit intitulé Suicide dans lequel il évoque le suicide, il y a plus de vingt ans, d'un ami cher, un ami d'enfance et de jeunesse, qui s'est tué à l'âge de 25 ans.

On se trouve donc devant un texte posthume qui nous trouble profondément et nous laisse perplexe, car on se demande s'il faut le considérer comme un message rétrospectif, une chronique d'une mort annoncée, une œuvre littéraire qui permet à l'auteur de survivre à sa mort, ou encore comme un récit qui éclaire le personnage et le suicide d'Édouard Levé.

Pour raconter l'ami suicidé, Levé s'adresse à lui directement et utilise le tu. Dès les premières lignes, il nous jette le suicide de son ami en pleine face:

Un samedi au mois d'août, tu sors de chez toi en tenue

de tennis accompagné de ta

femme. Au milieu du jardin, 
tu lui fais remarquer que tu as oublié ta raquette à la maison. Tu retournes la chercher, [...] Ta femme [...] elle est restée dehors, il fait beau, elle profite du soleil. Quelques instants plus tard, elle entend la décharge d'une arme à feu. Tu t'es tiré une balle dans la tête avec le fusil que tu avais soigneusement préparé.

Sur un ton froid et distancié, le narrateur va brosser le portrait de l'ami suicidé, qui est à la fois présent au monde et détaché de lui. Levé le présente comme un être stoïque, délicat et sensible, ne voulant pas décevoir, parlant peu, regardant et écoutant beaucoup les autres. S'il lui était facile de rencontrer des gens nouveaux en tête-à-tête, il lui était, par contre, difficile d'en rencontrer en groupe. II parlait peu, mais avec précision, et avec passion lorsque son interlocuteur lui était familier. Pas misanthrope, mais pas mondain non plus, il savait dialoguer avec n'importe qui, et préférait le mode de l'interrogation à celui de l'affirmation.

Ailleurs, le narrateur relève le côté solitaire de l'ami suicidé: il aimait se promener seul dans des villes inconnues, naviguer en solitaire, s'enfermer dans sa chambre. Cependant, d'autres aspects plus sociables tempèrent ce côté solitaire, puisqu'il aimait aussi jouer de la batterie et le faisait avec virtuosité, et qu'il nageait, courait, montait à cheval, jouait avec ses amis, au tennis, au squash et au ping-pong.

Le narrateur précise que l'ami suicidé avait une famille, des amis, une maison, qu'il ne manquait pas d'argent et n'était ni solitaire, ni pauvre, ni alcoolique. II réussissait le peu qu'il entreprenait car il choisissait de faire «peu mais bien, ou rien plutôt que mal ». Pourtant, il ne s'identifiait pas aux gens heureux, mais se projetait dans ceux qui avaient tout raté, ou rien réussi, comme les clochards.

Étant donné que le narrateur ne fait que décrire sans jamais analyser, le mystère du suicide de l'ami reste entier. En effet, le récit n'a pas pour but d'expliquer le suicide: "Expliquer ton suicide? Personne ne s'y est risqué. " II se contente de donner naissance à toutes sortes $d$ 'hypothèses sans fournir de réponses, bien qu'il donne quelques indices sur la présence d'un mal-être, au quotidien, qui pèse et étouffe «[...] impression de ne pas être soi en se regardant dans la glace, impression d'être ailleurs en étant quelque part, sensations irréelles [...] Jeune encore, ton désarroi était inconsolable parce que tu le jugeais infondé ».
Par contre, le narrateur parle abondamment de la mort choisie, préméditée, familière, qui n'est pas une négation de la vie, mais une affirmation d'un "goût pour l'inconnu en pariant que si, de l'autre côté, quelque chose existait, ce serait mieux qu'ici». On comprend qu'il s'agit d'une quête de bonheur qui va tellement loin qu'elle va audelà de la vie et prend le risque de n'y rien trouver. Notons que le narrateur nous met en garde contre la tentation d'expliquer toute une vie par le suicide, il nous dit que celui-ci ne doit pas devenir «le prisme essentiel, unique, à travers lequel tous les autres événements qui l'ont précédé doivent se lire, voire se comprendre."

Récit très troublant car au fur et à mesure qu'il avance, il nous semble qu'en fait le tu s'est substitué au je et que plus le narrateur nous parle de son ami, plus il nous parle de lui-même, de ce qu'il pense de la vie, de la mort et de sa propre mort volontaire à venir. En réalité, par le truchement de ce récit, il vit, par anticipation, sa mort et ses conséquences sur ses proches. Les similitudes avec l'ami suicidé étant trop nombreuses, il semblerait bien que Suicide nous mette en présence du double d'Édouard Levé. Soulignons notamment que les deux personnages ont tellement apprivoisé l'idée de leur mort qu'ils n'ont pas craint de franchir le pas qui les en séparait. De plus, tous deux n'ont jamais fait de tentatives de suicide ratées et ont, par contre, planifié leur mort jusque dans les moindres détails, sans rien laisser au hasard; Édouard Levé est allé jusqu'à laisser, bien en évidence, des lettres adressées à sa femme et à ses amis.

En fait, il ne faudrait pas s'étonner de ce dédoublement puisque le thème et le trouble de l'identité faisaient déjà partie des préoccupations de Levé, comme en témoigne son œuvre photographique: en 1999, sa première série, Homonyme, est une suite de clichés d'hommes ayant le même nom que des auteurs célèbres comme Henri Michaux, Eugène Delacroix ou Yves Klein. Un autre travail photographique, qu'il a réalisé sur des villes homonymes américaines: Bagdad, Amsterdam, Rome, témoigne aussi d'une préoccupation semblable.

En écrivant Suicide, Levé nous annonce qu'il a fait le choix de faire venir sa mort et de l'accueillir plutôt que de l'attendre, d'affirmer son libre arbitre en fixant l'échéance de l'inéluctable. Cette mort volontaire ne l'a pas empêché d'éprouver des regrets pour la tristesse qu'il allait causer à ses proches. D'ailleurs, Levé leur laissera des lettres qui leur éviteront des interrogations sans réponses et des interprétations erronées. Remarquons que son souci de déculpabiliser les proches est déjà présent dans Suicide quand il précise: «Personne d'autre que toi ne pouvait te donner plus de goût pour la vie que pour la mort [...]".

Avec Suicide, un livre vraiment pas comme les autres, Levé s'interroge sur l'existence, prolonge cette vie qu'il a voulu abréger, puisque grâce à l'œuvre littéraire il ouvre la porte à toutes sortes $d$ 'hypothèses concernant sa vie et sa mort, se survit d'une certaine manière et "donne vie à la mort". L'auteur nous offre un récit beau, puissant, sans fioritures, d'une grande sobriété, qui dégage une angoisse métaphysique profonde. Ses phrases directes, nettes et incisives ne peuvent laisser le lecteur indifférent; le récit se termine par une suite de tercets, dont le dernier a un accent prémonitoire:

Le bonheur me précède,

La tristesse me suit,

La mort m'attend.

On ne peut que regretter la disparition prématurée d'une personnalité attachante et d'un être talentueux qui ne sera pas oublié de si tôt.

\section{Shahira El Moutei-Khalil}

\section{RIEFF, David \\ Mort d'une inconsolée Les derniers jours de Susan Sontag}

Paris, Climat, 2008, 182 p.

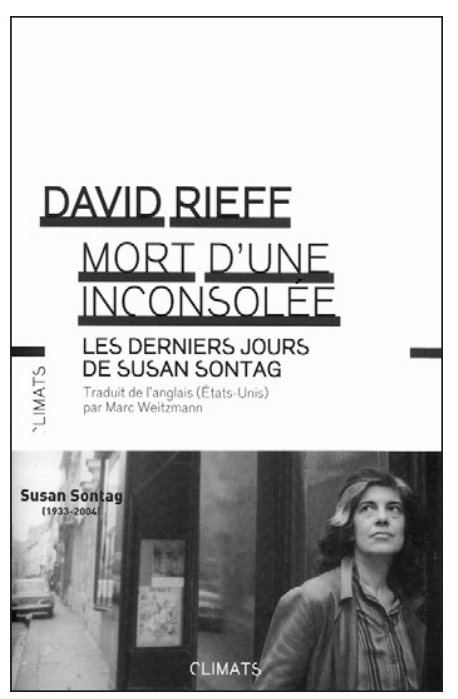

Une mère mourante, un fils présent. Elle, Susan Sontag, connue mondialement pour ses écrits (entre autres pour La maladie comme métaphore, Le sida et ses métaphores). Lui, David Rieff, célèbre journaliste couvrant les grands conflits internationaux (au New York Times Magazine entre autres) et renommé éditeur américain de Marguerite Yourcenar, Elias Canetti, Philippe Roth. Derrière ces personnages publics, une femme et un homme affrontent la mort: deux personnalités et un fort lien d'affection. En mai dernier, j'ai assisté à une entrevue que David Rieff donnait à un média. J'ai vu se présenter devant nous un homme dans la cinquantaine, très grand, élancé, au regard franc et direct, à la démarche lente et prudente: un fils venu raconter la mort et l'agonie de sa mère, un homme venu dire l'impuissance ressentie et l'immensité de sa perte. Un parcours dur mais exceptionnel; du 28 mars 2004 au 28 décembre 2004, neuf mois!

Susan Sontag connaît la maladie qu'est le cancer: en 1975 (début de la quarantaine), elle subit une mammectomie radicale après la découverte d'un cancer du sein à un stade avancé; en 1998, elle subit une chirurgie et une chimiothérapie suite à la découverte d'un sarcome utérin. Quand au début 2004 on lui annonce que la biopsie de la moelle épinière révèle, sans le moindre doute, un syndrome myélodysplasique (cancer du sang), elle a 71 ans. Le médecin, sans prononcer le mot "mort», suggère d'attendre que la maladie se déclare (une leucémie myéloïde) pour agir. «En clair, articula-t-elle, avec une circonspection poignante [...], il n'y a rien à faire. Rien que je puisse faire » (p. 21). Et le silence s'installe entre la mère et le fils. Les mots qui normalement auraient dû les unir étaient devenus inutiles, manquants, inadéquats. Deux ans après la mort de sa mère, David Rieff nous raconte la vie, la maladie et la mort: celle de sa mère, la sienne, la leur.

Susan Sontag a toujours aimé la vie. À 16 ans elle écrivait dans son Journal (dont Rieff prépare la publication) qu'elle était « incapable de seulement imaginer qu'un jour [elle] ne serait plus en vie» (p. 21). "Elle ne pouvait imaginer autre chose qu'être » (p. 26). Elle croyait en son destin, en "sa bonne étoile». Ses espoirs en tant qu'écrivain étaient tels qu'elle voulait vivre jusqu'à cent ans, "incapable d'envisager la perspective de son extinction" (p. 30). Il était impensable qu'elle disparaisse: "Elle ne pouvait que se projeter dans l'étape suivante" (p. 160). Toujours plus de temps, jamais rassasiée, émerveillée, glou- 